\title{
Round-off stability for multi-valued maps
}

\author{
Shyam Lal Singh ${ }^{1 *}$, Swami Nath Mishra ${ }^{2}$ and Sarika Jain ${ }^{3}$
}

\footnotetext{
*Correspondence: vedicmri@gmail. com

${ }^{1}$ Department of Mathematics, Pt. L. M. S. Govt. Postgraduate College (Autonomous), 21 Govind Nagar, Rishikesh 249201, India Full list of author information is available at the end of the article
}

\begin{abstract}
An iterative procedure for a map $T$ is said to be stable if the approximate sequence arising in numerical praxis converges to the point anticipated by the theoretical sequence. The study of stability of iterative procedures plays a vital role in computational analysis, game theory, computer programming, and fractal geometry. In generation of fractals, a sequence of approximations produces a stable set attractor only if the corresponding iterative procedure shows a stable behavior. The purpose of this article is to discuss stability of the Picard iterative procedure for a map $T$ satisfying Zamfirescu multi-valued contraction on a metric space.

MSC (2010): 47H10; 54H25; 65D15; 65D18.
\end{abstract}

Keywords: fixed point, stability of iterative procedures, Picard iterative procedure, Zamfirescu contraction, fractals

\section{Introduction}

Let $(X, d)$ be a metric space and $T: X \rightarrow X$. The solution of a fixed point equation $T x$ $=x$ for any $x \in X$, is usually approximated by a sequence $\left\{x_{n}\right\}$ in $X$ generated by an iterative procedure $f\left(T, x_{n}\right)$ that converges to a fixed point of $T$. However, in actual computations, we obtain an approximate sequence $\left\{y_{n}\right\}$ instead of the actual sequence $\left\{x_{n}\right\}$. Indeed, the approximate sequence $\left\{y_{n}\right\}$ is calculated in the following manner. First, we choose an initial approximation $x_{0} \in X$. Then we compute $x_{1}=f\left(T, x_{0}\right)$. But, due to rounding off or discretization of the function, we get an approximate value $y_{1}$, say, which is close enough to $x_{1}$, i.e., $y_{1} \approx x_{1}$. Consequently, when computing $x_{2}$, we actually compute $y_{2} \approx x_{2}$. In this way, we obtain an approximate sequence $\left\{y_{n}\right\}$ instead of the actual sequence $\left\{x_{n}\right\}$. The iterative procedure $f\left(T, x_{n}\right)$ is considered to be numerically stable if and only if the approximate sequence $\left\{y_{n}\right\}$ still converges to the desired solution of the equation $T x=x$. Urabe [1] initiated the study of this problem. The study of stability of iterative procedures plays a significant role in numerical mathematics due to chaotic behavior of functions and discretization of computations in computer programming. For a detailed discussion on the role of stability of iterative procedures, one may refer to Czerwik et al. [2,3], Harder and Hicks [4-6], Lim [7], Matkowski and Singh [8], Ortega and Rheinboldt [9], Osilike [10,11], Ostrowski [12], Rhoades [13,14], Rus et al. [15] and Singh et al. [16].

However, Ostrowski [12] was the first to obtain the following classical stability result on metric spaces.

Theorem 1.1. Let $(X, d)$ be a complete metric space and $T: X \rightarrow X$ a Banach contraction with contraction constant $q$, i.e., $d(T x, T y) \leq q d(x, y)$ for all $x, y \in X$, where 0 
$\leq q<1$. Let $p$ be the fixed point of $T$. Let $x_{0} \in X$ and $x_{n+1}=T x_{n}, n=0,1,2, \ldots$. Suppose that $\left\{y_{n}\right\}$ is a sequence in $X$ and $\varepsilon_{n}=d\left(y_{n+1}, T y_{n}\right)$. Then

$$
d\left(p, y_{n+1}\right) \leq d\left(p, x_{n+1}\right)+q^{n+1} d\left(x_{0}, y_{0}\right)+\sum_{j=0}^{n} q^{n-j} \varepsilon_{j} .
$$

Moreover, $\lim _{n} y_{n}=p$ if and only if $\lim _{n} \varepsilon_{n}=0$.

This result has found a respectable place in numerical analysis and computer programming and further extended by Harder and Hicks [5,6], Jachymski [17], Osilike $[10,11,18]$, Osilike and Udomene [19], Rhoades [13,14], Czerwik et al. [2] and Zhou [20].

The classical result on stability due to Ostrowski has been extended to multi-valued maps by Singh and Chadha [21] and further extended by Singh and Bhatnagar [22] and Singh et al. [23].

Furhter, stability of iterative procedures has a remarkable importance in fractal graphics while generating fractals. Its usefulness lies in the fact that in fractal graphics, fractal objects are generated by an infinite recursive process of successive approximations. An itertive procedure produces a sequence of results and tends towards one final object called a set attractor (fractal), which is independent of the initial choice. This stable character of set attractor is due to the stability of iterative procedure, else the system of underlying successive approximations would show chaotic behavior and never settle into a stationary state. However, fractals themselves have a variety of applications in digital imaging, mobile computing, architecture and construction, various branches of engineering and applied sciences. For recent potential applications of fractal geometry in related fields, one may refer to Batty and Longley [24], Buser et al. [25], Lee and Hsieh [26], Mistakeidis and Panagouli [27], Shaikh et al. [28] and Zmes$\mathrm{kal}$ et al. [29]. For connections of the round-off stability with the concept of limit shadowing for a fixed point problem involving multi-valued maps, one may refer to Petrusel and Rus [30].

The purpose of this article is to discuss the stability of Picard iterative procedure, i.e., $x_{n+1} \in f\left(T, x_{n}\right)=T x_{n}$ for a map $T$ satisfying Zamfirescu multi-valued contraction (cf. Definition 2.2).

\section{Preliminaries}

This section is primarily devoted to notations and definitions to be used in the sequel.

\subsection{Multivalued contractions}

Let $(X, d)$ be a metric space and

$C B(X)=\{A: A$ is a nonempty closed bounded subset of $X\}$,

$C L(X)=\{A: A$ is a nonempty closed subset of $X\}$.

For $A, B \in C L(X)$ and $\varepsilon>0$,

$$
\begin{aligned}
N(\varepsilon, A) & =\{x \in X: d(x, a)<\varepsilon \text { for some } a \in A\}, \\
E_{A, B} & =\{\varepsilon>0: A \subseteq N(\varepsilon, B), B \subseteq N(\varepsilon, A)\}, \\
H(A, B) & =\left\{\begin{array}{cc}
\text { inf } E_{A, B} \text { if } E_{A, B} \neq \phi \\
+\infty & \text { if } E_{A, B}=\phi .
\end{array}\right.
\end{aligned}
$$


$H$ is called the generalized Hausdorff metric (resp. Hausdorff metric) for $C L(X)$ (resp. $C B(X)$ ) induced by $d$. For any nonempty subsets $A, B$ of $X, d(A, B)$ will denote the gap between the subsets $A$ and $B$, while we write $d(a, B)$ for $d(A, B)$ when $A=\{a\}$.

An orbit $O\left(x_{0}\right)$ of a multi-valued map $T$ at a point $x_{0}$ is a sequence $\left\{x_{n}: x_{n} \in T x_{n-1}, n\right.$ $=1,2, \ldots\}$. For a single-valued map $T$, this orbit is $\left\{x_{n}: x_{n}=T x_{n-1}, n=1,2, \ldots\right\}$. A space $X$ is said to be $T$-orbitally complete [31,32] if every Cauchy sequence which is contained in $O\left(x_{0}\right)$ for some initial point $x_{0} \in X$ converges in $X$.

The study of fixed point theorems for multi-valued contractions was initiated by Markin [33] and Nadler [34]. The notion of multi-valued contractions have been generalized by many authors. For a good discussion on fixed point theorems for multivalued contractions, one may refer to Ćirić [31,32], Czerwik [35,36], Neammanee and Kalwkhao [37] and Rus and Petrusel [15,30]. However for the sake of comparison, we consider the following three conditions.

Let $(X, d)$ be a complete metric space and let $T: X \rightarrow C L(X)$. Then

Definition 2.1. (Nadler [34,38])

A map T: $X \rightarrow C L(X)$ is called a Nadler multi-valued contraction if

$$
H(T x, T y) \leq q d(x, y)
$$

for all $x, y \in X$, where $0 \leq q<1$.

Definition 2.2. (Zamfirescu [39])

A map $T: X \rightarrow C L(X)$ is called a Zamfirescu multi-valued contraction if there exist real numbers $\alpha, \beta$, and $\gamma$ satisfying $0 \leq \alpha<1,0 \leq \beta<\frac{1}{2}$ and $0 \leq \gamma<\frac{1}{2}$ such that for each $x, y \in X$ at least one of the following is true:

(i) $H(T x, T y) \leq \alpha d(x, y)$,

(ii) $H(T x, T y) \leq \beta[d(x, T x)+d(y, T y)]$,

(iii) $H(T x, T y) \leq \gamma[d(x, T y)+d(y, T x)]$.

Definition 2.3. (Ćirić [31])

A map T: $X \rightarrow C L(X)$ is called a Cirić generalized multi-valued contraction if there exists a nonnegative number $q$ such that

$$
H(T x, T y) \leq q \max \left\{d(x, y), d(x, T x), d(y, T y), \frac{1}{2}(d(x, T y)+d(y, T x))\right\}
$$

for all $x, y \in X$.

We remark that a Nadler multi-valued contraction ( $c f$. Definition 2.1) is continuous, while $T$ in Definition 2.2 need not be continuous. If we take $T: X \rightarrow X$, then (i) Definition 2.1 is the classical Banach contraction, (ii) Definition 2.2 is due to Zamfirescu [39] and (iii) Definition 2.3 is due to Ćirić [40]. In a comprehensive comparison of contractive maps, Rhoades [41] has listed 25 conditions for a single-valued map in a metric space. We remark that, for $T: X \rightarrow X$, the conditions given in Definition 2.1, 2.2, and 2.3 are respectively the conditions (1), (19), and (21'). For a comparison of contractive conditions for single valued maps more general than (21'), one may refer to Park [42] and see also Sessa and Cho [43]. Evidently, Nadler multi-valued contraction $\Rightarrow$ Zamfirescu multi-valued contraction $\Rightarrow$ Ćirić generalized multi-valued contraction.

We cite the following result due to Cirić [31].

Theorem 2.1. Let $T: X \rightarrow C L(X)$ be a Ćirić generalized multi-valued contraction such that $X$ is T-orbitally complete. Then: 
(i) for each $x_{0} \in X$, there exists an orbit $\left\{x_{n}\right\}$ of $T$ at $x_{0}$ and a point $p \in X$ such that $\lim _{n} x_{n}=p$;

(ii) $p \in T p$.

2.2 Stability of multivalued operators

Let $X$ be a metric space and $T: X \rightarrow C L(X)$. For a point $x_{0} \in X$, let

$$
x_{n+1} \in f\left(T, x_{n}\right)
$$

denote some iteration procedure. Let the sequence $\left\{x_{n}\right\}$ be convergent to a fixed point $p$ of $T$. Let $\left\{y_{n}\right\}$ be an arbitrary sequence in $X$ and set

$$
\varepsilon_{n}=H\left(y_{n+1}, f\left(T, y_{n}\right)\right), n=0,1,2, \ldots
$$

If $\lim _{n} \varepsilon_{n}=0$ implies that $\lim _{n} y_{n}=p$ then the iteration process defined in (2.2) is said to be $T$-stable or stable with respect to $T$ ( $c f$. [21]).

Ostrowski's stablity theorem [12] says that Picard iterative procedure for (singlevalued) Banach contraction is stable. Following is the extension of this theorem to multivalued contractions given by Singh and Chadha [21].

Theorem 2.2. Let $X$ be a complete metric space and $T: X \rightarrow C L(X)$ such that the condition given in Definition 2.1 holds for all $x, y \in X$. Let $x_{0}$ be an arbitrary point in $X$ and $\left\{x_{n}\right\}_{n=1}^{\infty}$ an orbit for $T$ at $x_{0}$ such that $\left\{x_{n}\right\}_{n=1}^{\infty}$ is convergent to a fixed point $p$ of $T$. Let $\left\{y_{n}\right\}$ be a sequence in $X$, and set

$$
\varepsilon_{n}=H\left(y_{n+1}, T y_{n}\right), n=0,1,2, \ldots
$$

Then

$$
d\left(p, y_{n+1}\right) \leq d\left(p, x_{n+1}\right)+q^{n+1} d\left(x_{0}, y_{0}\right)+\sum_{j=0}^{n} q^{n-j} \varepsilon_{j} .
$$

Further, if Tp is singleton then

$$
\lim _{n} y_{n}=p \text { if and only if } \lim _{n} \varepsilon_{n}=0 .
$$

We shall need the following result.

Lemma 2.1. (Harder and Hicks [6])

If $c$ is a real number such that $0<|c|<1$ and $\left\{b_{k}\right\}_{k=0}^{\infty}$ is a sequence of real numbers such that $\lim _{k \rightarrow \infty} b_{k}=0$, then $\lim _{n \rightarrow \infty}\left(\sum_{k=0}^{n} c^{n-k} b_{k}\right)=0$.

\section{Main results}

Theorem 3.1. Let $X$ be a complete metric space and $T: X \rightarrow C L(X)$ a Zamfirescu multi-valued contraction (cf. Definition 2.2). Let $x_{0}$ be an arbitrary point in $X$ and $\left\{x_{n}\right\}_{n=1}^{\infty}$ an orbit for $T$ at $x_{0}$ such that $\left\{x_{n}\right\}_{n=1}^{\infty}$ is convergent to a fixed point $p$ of $T$. Let $\left\{y_{n}\right\}_{n=1}^{\infty}$ be a sequence in $X$ and set $\varepsilon_{n}=H\left(y_{n+1}, T y_{n}\right), n=0,1,2, \ldots$. Then

(I) $d\left(p, y_{n+1}\right) \leq d\left(p, x_{n+1}\right)+\sum_{k=0}^{n} 2 \delta^{n+1-k} H\left(x_{k}, T x_{k}\right)+\delta^{n+1} d\left(x_{0}, y_{0}\right)+\sum_{k=0}^{n} \delta^{n-k} \varepsilon_{k}$,

where $\delta=\max \left\{\alpha, \frac{\beta}{1-\beta}, \frac{\gamma}{1-\gamma}\right\}$ and $n=0,1, \ldots$.

Further, if Tp is singleton then 
(II) $\lim _{n \rightarrow \infty} y_{n}=p_{\text {if }}$ and only if $\lim _{n \rightarrow \infty} \varepsilon_{n}=0$.

Proof: Let $x, y \in X$. Since $T$ is a Zamfirescu multi-valued contraction, $T$ satisfies one of (i), (ii), and (iii). If (ii) holds, then

$$
\begin{aligned}
H(T x, T y) & \leq \beta[d(x, T x)+d(y, T y)] \\
& \leq \beta[H(x, T x)+H(y, T y)] \\
& \leq \beta[H(x, T x)+H(y, x)+H(x, T x)+H(T x, T y)] \\
& =\beta[2 H(x, T x)+d(y, x)+H(T x, T y)] .
\end{aligned}
$$

So,

$$
H(T x, T y) \leq \frac{2 \beta}{1-\beta} H(x, T x)+\frac{\beta}{1-\beta} d(x, y) .
$$

If (iii) holds, then

$$
\begin{aligned}
H(T x, T y) & \leq \gamma[d(x, T y)+d(y, T x)] \\
& \leq \gamma[H(x, T y)+H(y, T x)] \\
& \leq \gamma[H(x, T x)+H(T x, T y)+H(y, x)+H(x, T x)] \\
& =\gamma[2 H(x, T x)+d(x, y)+H(T x, T y)]
\end{aligned}
$$

that is

$$
H(T x, T y) \leq \frac{2 \gamma}{1-\gamma} H(x, T x)+\frac{\gamma}{1-\gamma} d(x, y) .
$$

Thus at least one of the following is true for any $x, y \in X$ :

(i) $H(T x, T y) \leq \alpha d(x, y)$,

(ii) $H(T x, T y) \leq \frac{2 \beta}{1-\beta} H(x, T x)+\frac{\beta}{1-\beta} d(x, y)$.

(iii) $H(T x, T y) \leq \frac{2 \gamma}{1-\gamma} H(x, T x)+\frac{\gamma}{1-\gamma} d(x, y)$.

Let $\delta=\max \left\{\alpha, \frac{\beta}{1-\beta}, \frac{\gamma}{1-\gamma}\right\}$. Then

(i*) $H(T x, T y) \leq 2 \delta H(x, T x)+\delta d(x, y)$

for any $x, y \in X$.

Let $n$ be a nonnegative integer. Since

$$
d\left(p, y_{n+1}\right) \leq d\left(p, x_{n+1}\right)+d\left(x_{n+1}, y_{n+1}\right),
$$

we have

$$
\begin{aligned}
d\left(x_{n+1}, y_{n+1}\right) & \leq H\left(T x_{n}, y_{n+1}\right) \\
& \leq H\left(T x_{n}, T y_{n}\right)+H\left(T y_{n}, y_{n+1}\right) \\
& \leq 2 \delta H\left(x_{n}, T x_{n}\right)+\delta d\left(x_{n}, y_{n}\right)+\varepsilon_{n} .
\end{aligned}
$$

Consequently

$$
d\left(x_{n}, y_{n}\right) \leq 2 \delta H\left(x_{n-1}, T x_{n-1}\right)+\delta d\left(x_{n-1}, y_{n-1}\right)+\varepsilon_{n-1} .
$$


Therefore using (3.2) and (3.3) in (3.1), we obtain

$$
\begin{aligned}
d\left(p, y_{n+1}\right) \leq & d\left(p, x_{n+1}\right)+2 \delta H\left(x_{n}, T x_{n}\right)+\varepsilon_{n} \\
& +\delta\left[2 \delta H\left(x_{n-1}, T x_{n-1}\right)+\delta d\left(x_{n-1}, y_{n-1}\right)+\varepsilon_{n-1}\right] \\
= & d\left(p, x_{n+1}\right)+2\left[\delta H\left(x_{n}, T x_{n}\right)+\delta^{2} H\left(x_{n-1}, T x_{n-1}\right)\right] \\
& +\delta^{2} d\left(x_{n-1}, y_{n-1}\right)+\left(\varepsilon_{n}+\delta \varepsilon_{n-1}\right) .
\end{aligned}
$$

Repeat this process $(n-1)$ times to obtain

$$
d\left(p, y_{n+1}\right) \leq d\left(p, x_{n+1}\right)+\sum_{k=0}^{n} 2 \delta^{n+1-k} H\left(x_{k}, T x_{k}\right)+\delta^{n+1} d\left(x_{0}, y_{0}\right)+\sum_{k=0}^{n} \delta^{n-k} \varepsilon_{k} .
$$

This proves (I).

By $\left(i^{*}\right)$, we have

$$
\begin{aligned}
\varepsilon_{n} & =H\left(y_{n+1}, T y_{n}\right) \\
& \leq d\left(y_{n+1}, p\right)+H(p, T p)+H\left(T p, T y_{n}\right) \\
& \leq d\left(y_{n+1}, p\right)+H(p, T p)+2 \delta H(p, T p)+\delta d\left(p, y_{n}\right) .
\end{aligned}
$$

This yields $\varepsilon_{n} \rightarrow 0$ as $n \rightarrow \infty$, since $T p=\{p\}$ by hypothesis.

Conversly, suppose that $\varepsilon_{n} \rightarrow 0$ as $n \rightarrow \infty$.

First, we assert that $\lim _{k \rightarrow \infty} H\left(x_{k}, T x_{k}\right)=0$, if $T p=\{p\}$. For

$$
H\left(x_{k}, T x_{k}\right) \leq H\left(x_{k},\{p\}\right) \leq d\left(x_{k},\{p\}\right)+H\left(T p, T x_{k}\right) .
$$

Since $T$ is a Zamfirescu multi-valued contraction, it follows from (i), (ii), and (iii), (cf. Definition 2.2), that $\left\{T x_{k}\right\}$ is a Cauchy sequence. Consequently, $T x_{k} \rightarrow T p$ as $k \rightarrow \infty$. So making $k \rightarrow \infty,(3.4)$ yields the assertion.

Note that $0 \leq \delta<1$.

If $\delta=0$, then (I) yields $\lim _{n \rightarrow \infty} y_{n}=p$. So assume that $0<\delta<1$.

Then $\delta^{n+1} d\left(x_{0}, y_{0}\right) \rightarrow 0$ as $n \rightarrow \infty$.

Since $\lim _{k \rightarrow \infty} H\left(x_{k}, T x_{k}\right)=0, \lim _{k \rightarrow \infty} \varepsilon_{k}=0$. Therefore, by Lemma (2.1),

$$
\sum_{k=0}^{n} 2 \delta^{n+1-k} H\left(x_{k}, T x_{k}\right) \rightarrow 0 \text { and } \sum_{k=0}^{n} \delta^{n-k} \varepsilon_{k} \rightarrow 0 \text { as } n \rightarrow \infty .
$$

Hence from (I), $\lim _{n \rightarrow \infty} y_{n}=p$

We remark that the second term on the right-hand side of the conclusion (I) vanishes when $\beta=\gamma=0$. So we have the following.

Corollary 3.1. Theorem 2.2.

Proof: It comes from Theorem 3.1 when $\alpha=q$ and $\beta=\gamma=0$.

Corollary 3.2. (Harder and Hicks [6])

Let $(X, d)$ be a complete metric space and let $T: X \rightarrow X$ be a Zamfirescu contraction. Let $p$ be the fixed point of $T$. Let $x_{0} \in X$, and put $x_{n+1}=T x_{n}$ for $n=0,1,2, \ldots$, so that $\lim _{n \rightarrow \infty} x_{n}=p$. Let $\left\{y_{n}\right\}_{n=0}^{\infty}$ be a sequence in $X$ and set $\varepsilon_{n}=d\left(y_{n+1}, T y_{n}\right), n=0,1,2, \ldots$. Then 
(Ia) $d\left(p, y_{n+1}\right) \leq d\left(p, x_{n+1}\right)+\sum_{k=0}^{n} 2 \delta^{n+1-k} d\left(x_{k}, x_{k+1}\right)+\delta^{n+1} d\left(x_{0}, y_{0}\right)+\sum_{k=0}^{n} \delta^{n-k} \varepsilon_{k}$, where $\delta=\max \left\{\alpha, \frac{\beta}{1-\beta}, \frac{\gamma}{1-\gamma}\right\}$ and $n=0,1, \ldots$

Further, if $T p$ is singleton then

(IIa) $\lim _{n \rightarrow \infty} y_{n}=p$ if and only if $\lim _{n \rightarrow \infty} \varepsilon_{n}=0$.

Proof: It is exactly derivable from Theorem 3.1 when $\varepsilon_{n}=H\left(y_{n+1}, T y_{n}\right)=d\left(y_{n+1}, T y_{n}\right)$ when $T$ is single valued. Further, $H\left(x_{n}, T x_{n}\right)=d\left(x_{n}, x_{n+1}\right)$, if the map $T$ is single-valued.

We remark that $p \in X$ in (II) of Theorem 3.1, is not required to be the unique fixed point of $T$. The related condition emphasizes that $T p$ contains just one point.

The following, due to an idea of Singh and Whitfield [[44], p. 226] and Singh and Chadha [[21], p. 190], is another extension of Corollary 3.1.

Theorem 3.2. Let all the hypotheses of Theorem 3.1 hold, wherein the definition of $\varepsilon_{n}$ is replaced as follows:

$$
\varepsilon_{n}=d\left(y_{n+1}, p_{n}\right), p_{n} \in T y_{n}, n=0,1,2 \ldots
$$

Then

$$
d\left(p, y_{n+1}\right) \leq d\left(p, x_{n+1}\right)+\sum_{k=0}^{n} 2 \delta^{n+1-k} H\left(x_{k}, T x_{k}\right)+\delta^{n+1} d\left(x_{0}, y_{0}\right)+\sum_{k=0}^{n} \delta^{n-k}\left(\varepsilon_{k}+H_{k}\right),
$$

where $H_{k}=H\left(x_{k+1}, T x_{k}\right)$.

Further, if Tp is singleton then

(IVa) $\lim _{n \rightarrow \infty} y_{n}=p$ then $\lim _{n \rightarrow \infty} \varepsilon_{n}=0$,

(IVb) If $T$ is continuous and $\lim _{n \rightarrow \infty} \varepsilon_{n}=0$ then $\lim _{n \rightarrow \infty} y_{n}=p$.

Proof: Since $T$ is Zamfirescu multi-valued contraction in Theorem 3.2, we recall by

(i*) the following property: $H\left(T x_{n}, T y_{n}\right) \leq 2 \delta H\left(x_{n}, T x_{n}\right)+\delta d\left(x_{n}, y_{n}\right)$

for any $x_{n}, y_{n} \in X$. Therefore for any nonnegative integer $n$,

$$
\begin{aligned}
d\left(x_{n+1}, y_{n+1}\right) \leq & d\left(x_{n+1}, p_{n}\right)+d\left(p_{n}, y_{n+1}\right) \\
\leq & H\left(x_{n+1}, T y_{n}\right)+\varepsilon_{n} \\
\leq & H\left(x_{n+1}, T x_{n}\right)+H\left(T x_{n}, T y_{n}\right)+\varepsilon_{n} \\
\leq & H_{n}+2 \delta H\left(x_{n}, T x_{n}\right)+\delta d\left(x_{n}, y_{n}\right)+\varepsilon_{n} \\
\leq & H_{n}+2 \delta H\left(x_{n}, T x_{n}\right)+\delta\left[H_{n-1}+2 \delta H\left(x_{n-1}, T x_{n-1}\right)\right. \\
& \left.+\delta d\left(x_{n-1}, y_{n-1}\right)+\varepsilon_{n-1}\right]+\varepsilon_{n} \\
\leq & \delta^{2} d\left(x_{n-1}, y_{n-1}\right)+2 \delta\left[H\left(x_{n}, T x_{n}\right)+\delta H\left(x_{n-1}, T x_{n-1}\right)\right] \\
& +\delta\left(H_{n-1}+\varepsilon_{n-1}\right)+\left(H_{n}+\varepsilon_{n}\right) .
\end{aligned}
$$

Inductively,

$$
d\left(x_{n+1}, y_{n+1}\right) \leq \sum_{k=0}^{n} 2 \delta^{n+1-k} H\left(x_{k}, T x_{k}\right)+\delta^{n+1} d\left(x_{0}, y_{0}\right)+\sum_{k=0}^{n} \delta^{n-k}\left(H_{k}+\varepsilon_{k}\right),
$$

and the relation (III) follows as in the proof of (I).

To prove (IVa), first assume that $y_{n} \rightarrow p$ as $n \rightarrow \infty$.

Then $\varepsilon_{n}=d\left(y_{n+1}, p_{n}\right) \leq H\left(y_{n+1}, T y_{n}\right)$.

This, as in proof of Theorem 3.1, gives $\lim _{n} \varepsilon_{n}=0$. 
Now assume that $\mathrm{T}$ is continuous and $\lim _{n} \varepsilon_{n}=0$. From (III),

$$
d\left(p, y_{n+1}\right) \leq d\left(p, x_{n+1}\right)+\sum_{k=0}^{n} 2 \delta^{n+1-k} H\left(x_{k}, T x_{k}\right)+\delta^{n+1} d\left(x_{0}, y_{0}\right)+\sum_{k=0}^{n} \delta^{n-k} t_{k}
$$

where $t_{k}=\left(\varepsilon_{k}+H_{k}\right)$. In view of the (corresponding part of the) proof of Theorem 3.1 , it is sufficient to show that the sequence $\left\{t_{k}\right\}$ is convergent to 0 . Since, by one of the assumptions, the sequence $\left\{\varepsilon_{k}\right\}$ is convergent to 0 , it is enough to show that $\left\{H_{n}\right\}$ is also convergent to 0 . Since $T$ is continuous,

$$
\lim _{n} H_{n}=\lim _{n} H\left(x_{n+1}, T x_{n}\right)=H(p, T p)=0 .
$$

This completes the proof. $\square$

Corollary 3.3. (Singh and Chadha [21, Theorem 3])

Let all the hypotheses of Theorem 2.2 hold, wherein the definition of $\varepsilon_{n}$ is replaced by the following

$$
\varepsilon_{n}=d\left(y_{n+1}, p_{n}\right), p_{n} \in T y_{n}, n=0,1,2 \ldots
$$

Then

$$
d\left(p, y_{n+1}\right) \leq d\left(p, x_{n+1}\right)+q^{n+1} d\left(x_{0}, y_{0}\right)+\sum_{k=0}^{n} q^{n-k}\left(H_{k}+\varepsilon_{k}\right)
$$

where $H_{k}=H\left(x_{k+1}, T x_{k}\right)$. Further, if Tp is singleton then

$$
\lim _{n} y_{n}=p \text { if and only if } \lim _{n} \varepsilon_{n}=0 .
$$

Proof: Recall that a Nadler multi-valued contraction is continuous. So it comes from the fact that Definition 2.1 implies Definition 2.2.

It seems interesting to answer the following

Question: Can one replace Zamfirescu multi-valued contraction in Theorems 3.1 and 3.2 by the Ćiric generalized multi-valued contraction?

\section{Acknowledgements}

The authors thank the referees for their appreciation, deep understanding of the subject and useful suggestions to improve upon the article. S. L. Singh acknowledges the support of the University Grants Commission, New Delhi under Emeritus Fellowship.

\section{Author details}

${ }^{1}$ Department of Mathematics, Pt. L. M. S. Govt. Postgraduate College (Autonomous), 21 Govind Nagar, Rishikesh 249201, India 2Department of Mathematics, Walter Sisulu University, Mthatha 5117, South Africa ${ }^{3}$ Department of Information Technology, Amity University, Noida 201301 India

\section{Authors' contributions}

All authors read and approved the final manuscript.

Competing interests

The authors declare that they have no competing interests.

Received: 1 April 2011 Accepted: 8 February 2012 Published: 8 February 2012

\section{References}

1. Urabe, M: Convergence of numerical iteration in solution of equations. J Sci Hirishima Univ Sér A. 19, $479-489$ (1956)

2. Czerwik, S, Dlutek, K, Singh, SL: Round-off stability of iteration procedures for operators in b-metric spaces. J Natur Phys Sci. 11, 87-94 (1997) 
3. Czerwik, S, Dlutek, K, Singh, SL: Round-off stability of iteration procedures for set-valued operators in b-metric spaces. J Natur Phys Sci. 15(1-2):1-8 (2001)

4. Harder, AM: Fixed point theory and stability results for fixed point iterative procedures. Ph.D. Thesis, Univ of MissouriRolla. (1987)

5. Harder, AM, Hicks, TL: A stable iteration procedure for non-expansive mappings. Math Japon. 33, 687-692 (1988)

6. Harder, AM, Hicks, TL: Stability results for fixed point iteration procedures. Math Japon. 33, 693-706 (1988)

7. Lim, TC: On fixed point stability for set-valued contractive mappings with applications to generalized differential equations. J Math Anal Appl. 110, $436-441$ (1985). doi:10.1016/0022-247X(85)90306-3

8. Matkowski, J, Singh, SL: Round-off stability of functional iterations on product spaces. Indian J Math. 39(3):275-286 (1997)

9. Ortega, JM, Rheinboldt, WC: Iterative Solution of Nonlinear Equations in Several Variables. Academic Press, New York (1970)

10. Osilike, MO: Stability results for fixed point iteration procedures. J Nigerian Math Soc. 14, 17-29 (1995)

11. Osilike, MO: Stability results for the Ishikawa fixed point iteration procedure. Indian J Pure Appl Math. 26, 937-945 (1995)

12. Ostrowski, AM: The round-off stability of iterations. Z Angew Math Mech. 47, 77-81 (1967). doi:10.1002/ zamm.19670470202

13. Rhoades, BE: Fixed point theorems and stability results for fixed point iteration procedures. Indian J Pure Appl Math. 21, 1-9 (1990)

14. Rhoades, BE: Fixed point theorems and stability results for fixed point iteration procedures-II. Indian J Pure Appl Math. 24, 697-703 (1993)

15. Rus, IA, Petrusel, A, Petrusel, G: Fixed Point Theory 1950-2000: Romanian Contributions. House of the Book of Science, Cluj-Napoca (2002)

16. Singh, SL, Mishra, SN, Chadha, V: Round-off stability of iterations on product spaces. C R Math Rep Acad Sci Canada. 16(3):105-109 (1994)

17. Jachymski, JR: An extension of A. Ostrowski's theorem on the round-off stability of iterations. Aequations Math. 53, 242-253 (1997). doi:10.1007/BF02215974

18. Osilike, MO: Iterative solution of nonlinear equations of f-strongly accretive type. J Math Anal Appl. 200, 259-271 (1996). doi:10.1006/jmaa.1996.0203

19. Osilike, MO, Udomene, A: Short proofs of stability results for fixed point iteration procedures for a class of contractive type mappings. Indian J Pure Appl Math. 30(12):1229-1234 (1999)

20. Zhou, HY: Stable iteration procedures for strong pseudocontractions and nonlinear equations involving accretive operators without Lipschitz assumption. J Math Anal appl. 230, 1-10 (1999). doi:10.1006/jmaa.1998.6108

21. Singh, SL, Chadha, V: Round-off stability of iterations for multivalued operators. C R Math Rep Acad Sci Canada. 17(5):187-192 (1995)

22. Singh, SL, Bhatnagar, C: Stability of iterative procedures for multivalued operators. Proc of the Conference on Recent Trends in Mathematics and Its Applications. pp. 25-34.Ind. Soc. Math. Math. Sci., Gorakhpur (2003)

23. Singh, SL, Bhatnagar, C, Hashim, AM: Round-off stability of Picard iterative procedure for multivalued operators. Nonlinear Anal Forum. 10(1):13-19 (2005)

24. Batty, M, Longley, P: Fractal cities: a geometry of form and function. Academic Press, London (1994)

25. Buser, $\mathrm{P}$, Tosan, E, Weinand, Y: Fractal Geometry and its applications in the field of construction. Available at: http:// 150.217.71.164/ambientlab2010/docs/105c06_project_plan.pdf

26. Lee, WL, Hsieh, KS: A robust algorithm for the fractal dimension of images and its applications to the classification of natural images and ultrasonic liver images. Signal Process. 90(6):1894-1904 (2010). doi:10.1016/j.sigpro.2009.12.010

27. Mistakeidis, ES, Panagouli, OK: Strength evaluation of retrofit shear wall elements with interfaces of fractal geometry Eng Struct. 24(5):649-659 (2002). doi:10.1016/50141-0296(01)00131-6

28. Shaikh, YH, Khan, AR, Pathan, JM, Patil, A, Behere, SH: Fractal pattern growth simulation in electrodeposition and study of the shifting of center of mass. Chaos, Solitons \& Fractals. 42(5):2796-2803 (2009). doi:10.1016/j.chaos.2009.03.192

29. Zmeskal, O, Vala, M, Weiter, M, Stefkova, P: Fractal-cantorian geometry of space-time. Chaos, Solitons \& Fractals. 42(3):1878-1892 (2009). doi:10.1016/j.chaos.2009.03.106

30. Petrusel, A, Rus, IA: The theory of a metric fixed point theorem for multivalued operators. Proc Ninth International Conference on Fixed Point Theory and its Applications, Changhua, Taiwan, July 16-22 (2009). pp. 161-175.Yokohama Publ (2011)

31. Ćirić, LB: Fixed points for generalized multi-valued contractions. Mat Vesnik. 9(24):265-272 (1972)

32. Cirić, LB: A generalization of Banach's contraction principle. Proc Am Math Soc. 45, 267-273 (1974)

33. Markin, JT: A fixed point theorem for set-valued mappings. Bull Am Math Soc. 74, 639-640 (1968). doi:10.1090/500029904-1968-11971-8

34. Nadler, SB Jr: Multivalued contraction mappings. Pacific J Math. 30, 475-488 (1969)

35. Czerwik, S: A fixed point theorem for a system of multi-valued transformations. Proc Am Math Soc. 55, 215-219 (1976)

36. Czerwik, S: Fixed point theorems and special solutions of functional equations. Silesian University, Katowice. 428, 1-83 (1980)

37. Neammanee, K, Kalwkhao, A: Fixed point theorems of multi-valued Zamfirescu mapping. J Math Res. 2(2):150-156 (2010)

38. Nadler, SB Jr: Hyperspaces of Sets. Marcel-Dekker, New York (1978)

39. Zamfirescu, T: Fixed point theorems in metric spaces. Arch Math (Basel). 23, 292-298 (1972). doi:10.1007/BF01304884

40. Cirić, LB: Generalized contractions and fixed-point theorens. Publ Inst Math (Beograd) (NS). 12(26):19-26 (1971)

41. Rhoades, BE: A comparison of various definitions of contractive mappings. Am Math Soc. 226, 257-290 (1977)

42. Park, S: On general contractive type conditions. J Korean Math Soc. 17(1):131-140 (1980) 
43. Sessa, S, Cho, YJ: Compatible mappings and a common fixed point theorem of Chang type. Publ Math Debrecen. 43(34):289-296 (1993)

44. Singh, SL, Whitfield, JHM: Contractors and fixed points. Colloq Math. 55(2):219-228 (1988)

doi:10.1186/1687-1812-2012-12

Cite this article as: Singh et al:: Round-off stability for multi-valued maps. Fixed Point Theory and Applications 2012 2012:12.

Submit your manuscript to a SpringerOpen ${ }^{\circ}$ journal and benefit from:

- Convenient online submission

- Rigorous peer review

- Immediate publication on acceptance

- Open access: articles freely available online

- High visibility within the field

- Retaining the copyright to your article

Submit your next manuscript at $\gg$ springeropen.com 